\title{
Análisis comparativo de las normas de empaque y rotulado de fármacos veterinarios de la Comunidad Andina y la Unión Europea
}

\author{
Comparative Analysis of Standards packaging and labeling of Veterinary Drugs of the Andean Community \\ and the European Union
}

Francisco Jaramillo ${ }^{1}$, Arturo Clery ${ }^{2}$

\section{RESUMEN}

Este trabajo pretende ser una herramienta técnica, tanto para las autoridades competentes, como para el personal del área regulatoria de las empresas farmacéuticas veterinarias que necesiten registrar un producto veterinario en los bloques de las naciones europeas o andinas, describiendo los puntos en los que las normativas supranacionales son coincidentes y discrepantes, y de esta manera facilitar la conversión del formato de etiquetado y embalaje, dependiendo ante qué bloque se desea registrar el medicamento veterinario en cuestión.

PALABRAS CLAVE: empaque, rotulado, fármaco, Comunidad Andina, Unión Europea.

\section{SUMMARY}

This paper is a technical tool for both the competent authorities and staff of the regulatory area of veterinary pharmaceutical companies that need to register a veterinary product in blocks of European and Andean nations, describing the points where regulations supranational and discrepant coincide, and thus facilitate the conversion of labeling and packaging format, depending to which block you want to register the veterinary drug in question.

KEYWORDS: Packaging, labeling, drug, Andean Community, European Union.

\section{INTRODUCCIÓN}

Los países a lo largo de su historia han visto la conveniencia de formar bloques de manera voluntaria para lograr un mejor desarrollo económico y armonía en sus legislaciones. Ejemplo de estos bloques lo encontramos en la Comunidad Andina - CAN, la que según la Cancillería de la República de Colombia (2016) "es un mecanismo de integración subregional creado mediante el Acuerdo de Cartagena del 26 de mayo 1969, con el propósito de mejorar el nivel de vida y desarrollo equilibrado de los habitantes de los países miembros, mediante la integración y la cooperación económica y social”. Europa es quizás el caso más avanzado de unión supra estatal; el origen de la Unión Europea - UE (2016) "se encuentra en el periodo posterior a la Segunda Guerra Mundial. Sus primeros pasos consistieron en impulsar la cooperación económica con la idea de que, a medida que aumentara la interdependencia económica entre los países, disminuirían las posibilidades de conflicto".

Las relaciones inter bloques andino - europeo han sido muy profundas, y ya desde la primera etapa de relaciones birregionales en 1973, el sector agropecuario ha sido prioritario (Camacho y Molina, 2005).

Carrera de Medicina Veterinaria, Facultad de Ciencias de la Salud, Universidad de Las Américas. Quito, Ecuador.

Universidad Estatal Península de Santa Elena. La Libertad, Ecuador. 
Los países de la Unión Europea son importantes socios comerciales de los países que integran la CAN, esto hace que se puedan dar oportunidades para las farmacéuticas que busquen registrar sus productos en los dos bloques. Según Brown y Torres (2012) "en cuanto a los principales mercados, el comercio de la CAN está diversificado entre varias regiones. El principal socio comercial es Estados Unidos, seguido de la UE. Esta representa un mercado importante debido a que los productos andinos se colocan a precios muy atractivos"; vale recalcar que el comercio se da por separado entre los países que forman la CAN con los EEUU y con la UE, sin existir un acuerdo global CAN-UE o CAN-EEUU.

\section{METODOLOGÍA}

La presente investigación es de corte interpretativo y fundamentalmente está basada en el análisis documental, y por lo tanto en el estudio e interpretación del contenido o "discurso".

Se utilizó también el método bibliográfico documental, "como parte esencial de un proceso de investigación científica, constituyéndose en una estrategia donde se observa y reflexiona sistemáticamente sobre realidades, usando para ello diferentes tipos de documentos" (Cruz, 2012). Se indagó información publicada por la comunidad científica, garantizando la calidad de los fundamentos teóricos, concretando las nociones básicas, principios generales y requisitos principales.

Se aplicó adicionalmente el método exegético, es decir, la investigación se apoyó en la interpretación realizada por académicos, expertos e instituciones, sobre el contenido de los preceptos jurídicos y la exégesis del propio autor (Pérez, 2009).

Otro método utilizado fue el de concordancia y derecho comparado. El derecho comparado es una "disciplina dedicada al estudio del derecho extranjero, de ahí su denominación inicial, cuyo análisis y sistematización permiten acceder a las claves para un mejor conocimiento y comprensión del tema, aplicando un método de investigación propio, el método comparado" (Morán, 2002).

Asimismo, la hermenéutica justificó los métodos utilizados en la presente investigación. En este sentido, la hermenéutica "nos enseña las condiciones para entender el significado de las palabras y la intención de los textos" (Kaufmann, 1996).

\section{RESULTADOS}

\section{Respecto a normativas}

El Parlamento Europeo y el Consejo de la UE como órganos legislativos expiden el 06 de noviembre de 2001 la Directiva 2001/82/CE, por la que se establece un código comunitario sobre medicamentos veterinarios, que reagrupa varias directivas anteriores en un texto único. Esta Directiva forma parte de las normas aplicables a los medicamentos en la UE, referente a legislación farmacéutica de productos médicos de uso veterinario (figura 1).

Dentro de su organización o Sistema Andino de Integración, la CAN tiene varios estamentos, siendo uno de ellos la Comisión, la que teniendo capacidad legislativa expidió la Decisión 483 de 08 de junio del

\section{Unión Europea}

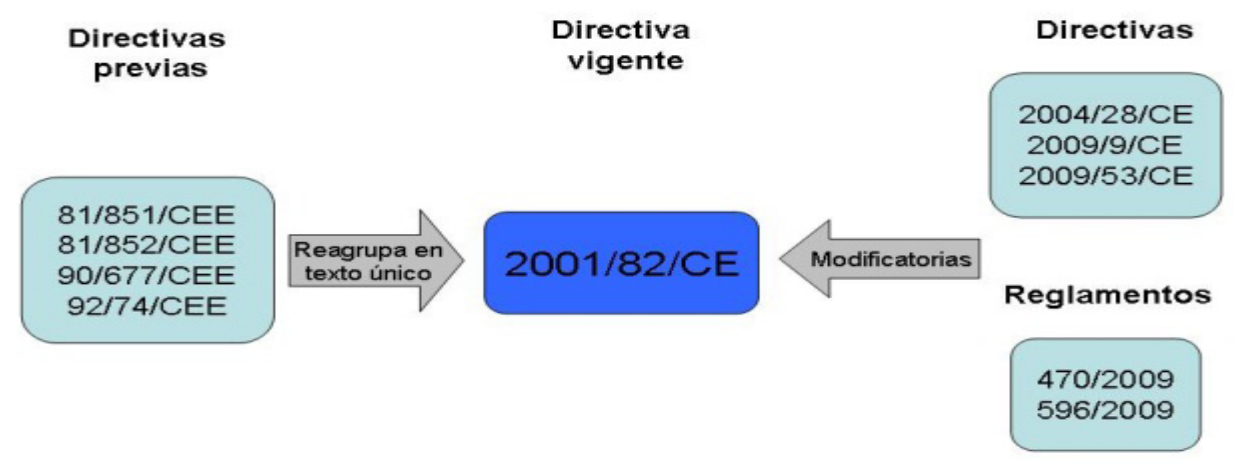

Figura 1. Normativa de la UE 
2000, normas para el registro, control, comercialización y uso de productos veterinarios. Esta Decisión es la primera legislación de la CAN en temas de registro de productos veterinarios, y armoniza los procedimientos de registro y control de productos veterinarios entre los países miembros de la Comunidad Andina (Calvo y Espino, 2002).

En 2011 se publica la Decisión 769 que sustituiría a la Decisión 483, sin embargo no se encuentra vigente por estar aún en discusión el manual técnico para su aplicación entre los países miembros de la CAN, razón por la que en el presente análisis se tomó a la Decisión 483 como la legislatura vigente de la CAN (figura 2).

En este sentido, el presente trabajo comparó la Directiva consolidada 2001/82/CE en su Título V con el Título VII de la Decisión 483 de la CAN, las que tienen que ver con las normas de empaque y rotulado de fármacos veterinarios.

\section{Respecto a la autoridad nacional competente}

La Decisión 483 designa a los Ministerios de Agricultura de cada país miembro, o a la entidad que sus gobiernos designen, como Autoridad Nacional Competente, responsables del cumplimiento de la misma.

La Directiva consolidada 2001/82/CE menciona a las autoridades competentes de los Estados miembros, como las habilitadas para las aprobaciones de solicitudes de autorización de comercialización de fármacos veterinarios, y se reserva para el procedimiento comunitario centralizado la competencia de la Agencia Europea de Evaluación de Medicamentos.

\section{Respecto a la terminología}

La Directiva 2001/82/CE emplea una terminología que es pertinente mencionar:

Acondicionamiento primario: el envase o cualquier otra forma de acondicionamiento que se encuentre en contacto directo con el medicamento.

Denominación común: la denominación común internacional recomendada por la Organización Mundial de la Salud (OMS), o en su defecto, la denominación común habitual.

Embalaje exterior: el embalaje en que se coloca el acondicionamiento primario.

Etiquetado: la información que figura en el embalaje exterior o en el acondicionamiento primario.

Medicamento veterinario: toda sustancia o combinación de sustancias (a) que se presente como poseedora de propiedades curativas o preventivas con respecto a las enfermedades animales; o (b) que pueda administrarse al animal con el fin de reestablecer, corregir o modificar las funciones fisiológicas del animal ejerciendo una acción farmacológica, inmunológica o metabólica, o de establecer un diagnóstico médico.

Medicamento homeopático veterinario: todo medicamento veterinario obtenido a partir de estancias denominadas cepas homeopáticas, con arreglo a un procedimiento de fabricación homeopático descrito en la farmacopea europea o, en su defecto, en las farmacopeas utilizadas en la actualidad de forma oficial en los Estados miembros. Un medicamento

\section{COMUNIDAD ANDINA DE NACIONES}

Decisión vigente

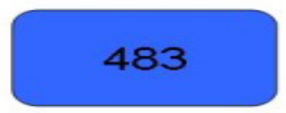

Decisión sustitutoria

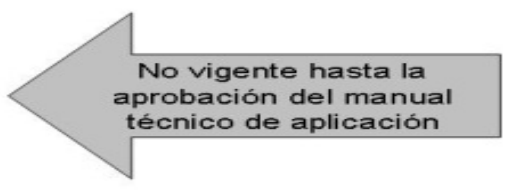

769

Figura 2. Normativa de la CAN 
homeopático veterinario podrá contener varias sustancias.

Prospecto: la nota informativa para el usuario, que acompaña al medicamento.

Las definiciones de la Decisión 483 para el tema de etiquetado son:

- Etiqueta: información impresa bajo cualquier sistema, que deben llevar los envases o empaques cualquiera que sea su material.

- Inserto, prospecto o folleto: impreso que tiene información complementaria a la consignada en la etiqueta de un producto veterinario.

- Rotulado: información impresa que consigna la etiqueta, caja e inserto o prospecto adjunto a los productos veterinarios.

- Alimento medicado: Alimentos que en su composición incluyen una sustancia anticoccidial, un promotor de crecimiento $u$ otros que sean aceptados por la Autoridad Nacional Competente

- Producto veterinario: Toda sustancia química, biológica, biotecnológica o preparación manufacturada cuya administración a los animales, en forma individual o colectiva, directamente o mezclado con los alimentos tiene como propósito la prevención, diagnóstico, curación o tratamiento de las enfermedades de los animales. Se incluye entre ellos a los aditivos, suplementos, promotores, mejoradores de la producción animal, antisépticos desinfectantes de uso ambiental o para desinfección de equipos, y pesticidas y todo otro producto que, utilizado en los animales y su hábitat, restaure o modifique las funciones orgánicas y fisiológicas, cuide y proteja sus condiciones de vida. Comprende también los productos destinados al embellecimiento de los animales.

\section{Respecto al empaque y rotulado de la directiva $2001 / 82 / C E$}

Recalca que tanto el acondicionamiento primario como el embalaje exterior deberán ser aprobados por la autoridad competente, y contener información concordante con la ficha técnica del producto.

Si un producto no tiene embalaje exterior, todos los datos deberán indicarse en el acondicionamiento primario.

Por la naturaleza de la UE, se estipula que los datos sobre especie de destino, vía de administración, tiempo de espera en animales de producción, caducidad, precauciones y advertencias, y las indicaciones "de uso veterinario" o "de uso veterinario - medicamento sujeto a prescripción veterinaria" entre otros, deberán ser redactadas tanto en la caja como en el recipiente en la o las lenguas del país de comercialización. El contenido en peso o volumen se debe señalar en el envase solamente.

La Directiva pide señalar las precauciones a tomarse en la eliminación de los restos y residuos de los medicamentos, y una referencia a los posibles sistemas de recogida de los mismos.

Para las especies productoras de alimentos, el tiempo de espera debe estar claramente señalado, inclusive en los que tienen un tiempo de espera de cero.

La Directiva permite que los Estados miembros autoricen o exijan más información, siempre que no sea contradictoria con el derecho comunitario, para ello se dispone de una zona separada de la requerida a nivel comunitario.

Esta Directiva hace una diferenciación para las ampollas y acondicionamientos primarios pequeños de una sola dosis. Señala un número mínimo de datos que deben figurar en las ampollas permitiendo que los demás datos figuren en el embalaje exterior, y en el caso de acondicionamientos pequeños de una dosis en los que es imposible imprimir los datos, los mismos serán impresos en el embalaje exterior.

Respecto al prospecto, deberá estar adjuntado de manera obligatoria, a menos que toda la información exigida figure en el acondicionamiento primario o en el embalaje exterior. Podrá estar redactado en varias lenguas siempre que una de estas sea la lengua oficial del estado donde se comercializa el producto, que contenga toda la información y que esta información sea idéntica en cada una de estas lenguas. Si el producto va a ser administrado exclusivamente por veterinarios el prospecto puede obviar determinados datos o lenguas.

La Directiva no afecta a las disposiciones que cada Estado miembro tenga en cuanto a precio, condiciones de expendio y sobre la propiedad industrial del fármaco.

Los medicamentos homeopáticos deben seguir las mismas disposiciones, pero además deben tener 
Tabla 1. Características de empaque y rotulado de los fármacos veterinarios.

La Decisión 483 dispone:

El nombre del producto veterinario.

La fórmula o composición del producto veterinario, precisando el o los principios activos, declarados según la aprobación del registro.

Indicaciones de uso.

Número de registro y organismo otorgante. Nombre y dirección del establecimiento, fabricante, titular del registro, representante o importador, cuando corresponda.

Número de serie, lote o partida.

Fecha de vencimiento.

Dosis sugerida por especie, forma de administración e instrucciones de uso, indicando en forma notoria la leyenda "Uso Veterinario". En casos necesarios, la dosis podrá ser modificada a indicación expresa del médico veterinario.

Período de retiro, tiempo de espera o restricciones de uso, cuando corresponda.

Aspectos toxicológicos, advertencias, contraindicaciones o reacciones adversas y antídotos, si existiesen.

Condiciones de almacenamiento (temperatura y humedad si corresponden).

Declaración de venta bajo receta profesional cuando corresponda.

Indicación expresa de mantenerlo fuera del alcance de los niños.
La Directiva 2001/82/CE dispone:

La denominación del medicamento veterinario, seguida de su dosificación y forma farmacéutica. Se indicará la denominación común en caso de que el medicamento contenga sólo una sustancia activa y su denominación sea un nombre de fantasía.

La composición cualitativa y cuantitativa de sustancias activas por dosis o según la forma de administración, para un determinado volumen o peso, utilizando las denominaciones comunes.

El tiempo de espera para los medicamentos veterinarios que deban administrarse a animales productores de alimentos, para todas las especies afectadas y para los diferentes alimentos de que se trate (carne y despojos, huevos, leche, miel), incluidos aquellos cuyo tiempo de espera sea de cero.

El número de la autorización de comercialización. El nombre o la razón social y el domicilio o la sede social del titular de la autorización de comercialización, y en su caso, del representante del titular designado por este último.

El número del lote de fabricación.

La fecha de caducidad, en lenguaje comprensible.

La indicación «de uso veterinario» o respecto de los medicamentos a que se refiere el artículo 67, la indicación «de uso veterinario - medicamento sujeto a prescripción veterinaria».

Las precauciones especiales para eliminar los medicamentos no utilizados o los residuos derivados de los mismos, en su caso, así como una referencia a los posibles sistemas de recogida apropiados existentes.

Las especies animales a las que esté destinado el medicamento veterinario, el modo y en aquellos casos en que sea necesario, la vía de administración. Se deberá prever un espacio libre para indicar la posología prescrita.

Las precauciones particulares de conservación, si hubiera lugar.

No aplica.

No aplica. la leyenda "medicamento homeopático para uso veterinario".

Existe un procedimiento de registro simplificado para medicamentos homeopáticos, parte de sus requerimientos es que no figure la indicación terapéutica en la etiqueta (Fernández, 2010). De ser este el caso debe incluir la leyenda "medicamento homeopático veterinario sin indicaciones terapéuticas aprobadas".

\section{Respecto a empaque y rotulado de la Decisión 483}

Señala específicamente al español como el idioma en que se redactará la información sobre el producto. Junto a la dosis por especie, forma de administración e instrucciones, deberá constar la leyenda "uso veterinario".

La fórmula o composición debe precisar los principios activos declarados y los aspectos 
toxicológicos, contra indicaciones, reacciones adversas y antídotos, de ser el caso.

Si la autoridad nacional competente así lo estipula, se debe incluir la declaración de venta bajo receta profesional. Y siempre tendrá una indicación expresa de mantenerlo fuera del alcance de los niños, junto con los demás datos informativos del producto. Si por motivos de espacio físico no es posible incluir toda la información, se podrá incluirla en el folleto que deberá estar adjunto al producto en su expendio. Pero los aspectos toxicológicos, reacciones adversas y los riesgos ambientales y para las personas, así como la temperatura de almacenamiento en los casos que la necesite, deben constar tanto en la etiqueta como en el folleto adjunto.

La Decisión tiene un capítulo sobre las restricciones en el rotulado, en donde se precautela que en el etiquetado no se vaya a incluir información falsa o que lleve a cometer errores.

En el siguiente cuadro comparativo se aprecian las características de empaque y rotulado de los fármacos veterinarios de las dos normativas (tabla 1) :

\section{Respecto a las posibilidades de comercialización entre los países de ambos bloques}

La CAN y UE se otorgan en la actualidad mutuamente el trato de Nación Más Favorecida. Las relaciones comerciales entre la UE y la CAN se iniciaron con el Sistema de Preferencias Generalizadas (SPG), que permitió el acceso preferencial al mercado comunitario para los productos provenientes de países en vías de desarrollo.

La apertura de negociaciones del Acuerdo de Asociación UE-CAN se produjo durante la XVII Reunión de Presidentes de la región, celebrada en Tarija (Bolivia) en junio de 2007. Tras la celebración de tres rondas de negociación, las Partes decidieron implementar un nuevo sistema de negociación, mediante el cual se admitió que los países andinos que lo desearan podrían negociar, de forma independiente, la parte comercial del Acuerdo de Asociación.

En este escenario, la UE y la CAN empezaron las negociaciones para un Acuerdo de Libre Comercio en 2007. En marzo de 2010, la UE, Colombia y Perú concluyeron la negociación del nuevo Acuerdo Multipartito, bajo presidencia española, y dejaron constancia de ello en la Declaración Conjunta de la
VI Cumbre UE-América Latina y Caribe de mayo de 2010, celebrada en Madrid.

Este ambicioso acuerdo en su vertiente comercial se aplica con Perú y con Colombia desde el 2013. Los otros dos países miembros de la Comunidad Andina, Bolivia y Ecuador, mantuvieron la posibilidad de adherirse al Acuerdo si así lo deseaban, en base a lo cual se retomaron las negociaciones con Ecuador en 2014, que tras cuatro rondas, se acordó en el mes de julio, su incorporación al Acuerdo.

El Acuerdo permite beneficiarse de un mejor acceso de las principales exportaciones de la CAN a la UE (pesca, plátanos, frutas, flores, café, cacao), yendo los términos del nuevo acuerdo más allá del Sistema Generalizado de Preferencias concedido por la Unión Europea. Pero sobre todo, puede permitir la comercialización efectiva de fármacos veterinarios entre los países de estos bloques.

En cuanto a ventajas para la UE, el Acuerdo le proporciona un mejor acceso al mercado de los países de la CAN para muchas de sus exportaciones clave, por ejemplo en el sector automovilístico o de maquinarias. El Acuerdo incluye un ambicioso resultado sobre acceso al mercado de servicios, establecimiento y contratación pública.

Agradecimientos: Se deja constancia de agradecimiento a la colaboración académica de

la Universidad Estatal Península de Santa Elena UPSE, a través de su Observatorio de

Políticas Sociales.

\section{Correspondencia:}

Francisco Jaramillo

Correo: electrónico:francisco.jaramillo.cisneros@ udla.edu.ec

\section{REFERENCIAS BIBLIOGRÁFICAS}

1. Brown, A. \& Torres, P. (2012). La relación comercial Comunidad Andina- Unión Europea y la postura de Ecuador. Latinoamérica, Revista de estudios Latinoamericanos, 55, 75-99. Recuperado de: http://www.scielo.org.mx/scielo.php?script=sci arttext\&pid $=\mathrm{S} 1665-85742012000200004 \& \operatorname{lng}=\mathrm{es} \& \mathrm{t}$ $\operatorname{lng}=\mathrm{es}$

2. Calvo, A. \& Ospino, G. (2002). Implementacion de la documentación exigida por las buenas practicas de manufactura para la fabricación de medicamentos veterinarios. (Tesis de Especialización en Gerencia 
de Producción y Operaciones. Universidad de La Sabana, Bogotá, Colombia).

3. Camacho, G. H., \& Molina, S. (2005). IIRSA y la integración regional. OSAL, (17).

4. Cancillería de la República de Colombia. (2016). Comunidad Andina (CAN). Bogota: Cancillería de la República de Colombia Recuperado de: http:// www.cancilleria.gov.co/international/regional/can

5. Cruz, L. (2012). Metodología de la Investigación. Colima: Universidad Multitécnica Profesional.

6. Fernández, L. (2010). Bases para la implementación de una legislación nacional sobre medicamentos homeopáticos. (Tesina. Universidad de Belgrano, Buenos Aires, Argentina).
7. Kaufmann, A. (1996). Concepción hemenéutica del método jurídico. Persona y Derecho, 35, 11-36.

8. Morán, G. (2002). El derecho comparado como disciplina jurídica: la importancia de la investigación y la docencia del derecho comparado y la utilidad del método comparado en el ámbito jurídico. Anuario da Facultade de Dereito da Universidade da Coruña, 6, 501-530.

9. Pérez, A. (2009). El método en el derecho administrativo. Revista de Estudios Jurídicos Segunda Época, 9, 59-100.

10. Unión Europea. (2016). EUROPA - Qué es la UE. Ginebra: Unión Europea. Recueprado de: http:// europa.eu/about-eu/basic-information/about/index es.htm 